\title{
Takotsubo Cardiomyopathy Resulting from Transient Global Amnesia Following a Sexual Encounter
}

\section{Kamalpreet Dhaliwal, Sonia Randhawa, Nabihah Kabir, Yadwinder Sidhu, Harsimran Singh, Shawn Banash, Muhammed Janjua and Vib- hav Bansal*}

Department of Neurology, Mercy Health, Rockford, IL, USA

*Corresponding Author: Vibhav Bansal, Department of Neurology, Mercy Health, Rockford, IL, USA.
Received: April 15, 2020

Published: April 28, 2020

(C) All rights are reserved by Vibhav Bansal., et al.

\begin{abstract}
Background: Transient global amnesia (TGA) is a neurological condition described as acute onset anterograde amnesia that resolves within 24 hours. TGA is often associated with migraine history, psycho-physical stress, and cardiovascular risk factors. Takotsubo Cardiomyopathy (TTC), also known as Broken Heart Syndrome, is a reversible, stress-induced weakening of the left ventricle (LV) resulting in cardiomyopathy. Concurrent occurrence of the two syndromes have rarely been reported. To date, there have been 10 documented concurrent TGA and TTC cases, all unrelated to sexual triggers. Here we report the first patient with simultaneous TGA and TTC following a sexual encounter.

Insights and Aim: Summarize a case and its associated factors to establish awareness of a possible causal relationship between TGA and TTC following a sexual encounter as a precipitating factor.

Case Report: A 66 year-old woman presented to the emergency department with sudden onset confusion and forgetfulness witnessed by the patient's husband immediately after sexual intercourse.

Neurologic exam on admission revealed short-term memory deficits, resulting in repeated questions of similar nature regarding the peri-ictal period. Lab results revealed an elevated High Sensitivity Troponin-T of $51 \mathrm{ng} / \mathrm{L}$ (normal, < $14 \mathrm{ng} / \mathrm{L}$ ); all other pertinent labs were within normal limits. The patient's neurological manifestations of amnesia resolved 14 hours after the time of presentation. Conclusion: Although TGA is considered a primary neurologic disorder, it is important to recognize a possible relationship between TGA and TTC in order to manage the cardiac complications that may arise from TTC.
\end{abstract}

Keywords: Stress Cardiomyopathy; Broken Heart Syndrome; Transient Global Amnesia; Heart Failure

\section{Introduction}

Transient global amnesia (TGA) is a neurological condition characterized by acute onset anterograde amnesia resolving within 24 hours. It is self-limited with restoration of memory function without recollection of the details during the transient episode. It rarely recurs. During an episode, other cognitive functions are generally intact including language and comprehension. The patient often appears disorganized and perseverative, which should not be misconstrued as delirium. It is primarily seen in older females with a mean age of onset between 60 and 65 years.

Although the incidence of TGA has been estimated as 5.2 to 10 per 100,000 per year, the incidence of TGA in combination with Takotsubo cardiomyopathy (TTC) is rare [2].
TTC, also known as stress-induced cardiomyopathy, is characterized as a transient systolic dysfunction of the left ventricle, imitating myocardial infarction (MI) without the presence of angiographic evidence of obstructive coronary artery disease. The regional wall motion abnormalities extend beyond any territory perfused by a single epicardial coronary artery. Pathologically, the inflammatory changes in TTC are non-necrotic compared to that seen in myocardial infarction [4].

While TGA and TTC typically result in temporary dysfunction ultimately resolving without residual effects, there have been two reported cases of both TGA and TTC occurrence with long-term complications. These complications included a cerebellar stroke and persistent bradycardia requiring pacemaker implantation. Therefore, it is important to evaluate risk factors, triggers, comorbidities 
and plausible complications related to these two syndromes occurring simultaneously [8].

To date, there are only 10 documented cases of co-occurrence of TGA and TTC, none of which have had a sexual encounter as a possible trigger. Here we report a patient who presented with findings significant for TGA and TTC following a sexual encounter.

\section{Case Report}

A 66-year-old Caucasian female with a past medical history of post-menopausal hormonal deficiency and anxiety presented to the emergency department with sudden restlessness during sexual activity. Thereafter, the patient was noted to be acutely confused and forgetful. In the emergency department, the patient suffered from a panic attack.

On presentation, patient was noted to have tachycardia with a heart rate of 103 beats/minute, all other vital signs were within normal range. Neurologic exam on admission revealed short-term memory deficit resulting in repeated questions of similar nature, but otherwise was within normal limits with preservation of longterm memory.

Lab results revealed an elevated High Sensitivity Troponin-T of $304 \mathrm{ng} / \mathrm{L}$ (normal, < $14 \mathrm{ng} / \mathrm{L}$ ), all other pertinent labs were within normal limits. An Electrocardiogram was significant for ST depression in the lateral leads and ST segment elevation in the aVR lead (Figure 1).

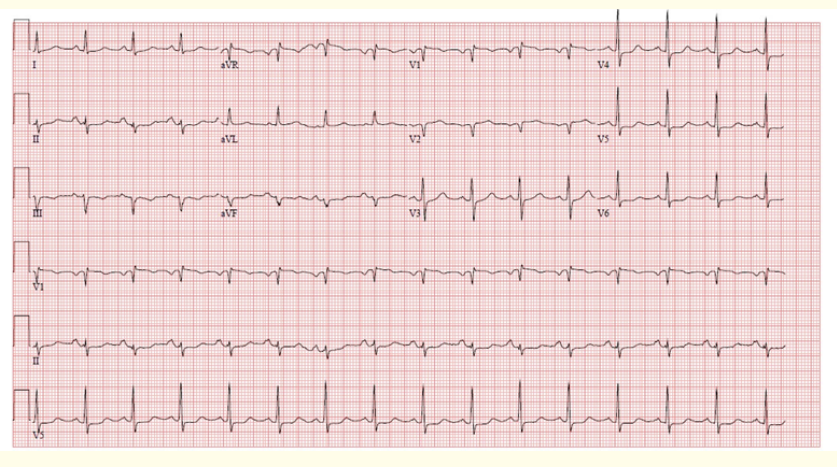

Figure 1: Electrocardiogram on admission. Normal sinus rhythm, Possible Left atrial enlargement, Left axis deviation.

Transthoracic echocardiography revealed a calculated Ejection Fraction (EF) of $40 \%$ along with wall motion abnormalities; specifically, there was antero-apical and lateral wall hypokinesis with good contractility in the bases suggestive of stress-induced cardiomyopathy (Figure 2).

The patient was started on a Heparin drip. On day 2 of hospitalization, patient underwent a coronary angiography that revealed normal coronary arteries without significant stenosis in any of the

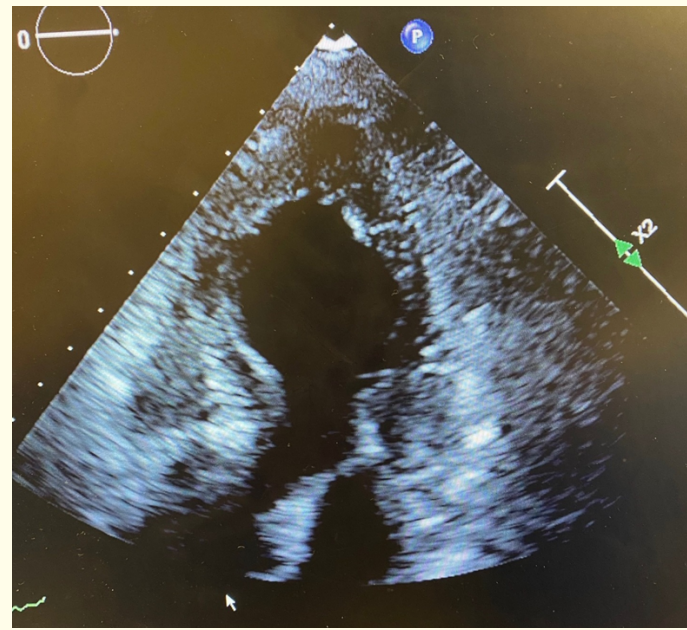

Figure 2: Echocardiographic findings of TTC: Normal left ventricle size noted. Ejection fraction is calculated at $40 \%$ with wall motion abnormalities. Antero apical and lateral wall hypokinesis with good contractility in the bases suggestive of stress-induced cardiomyopathy.

major vessels, an estimated EF of $40 \%$ with hypokinesis of the midanterior wall consistent with Takotsubo cardiomyopathy. An MRI of the brain was unremarkable. CTA of Head and Neck showed no significant abnormalities and was unremarkable.

The patient's neurological manifestations of amnesia resolved approximately 14 hours after the initial time of presentation. On day 4, the patient was discharged home on Aspirin $81 \mathrm{mg}$, Metoprolol $20 \mathrm{mg}$ BID and Atorvastatin $20 \mathrm{mg}$.

\section{Discussion}

Transient global amnesia (TGA) and Takotsubo Cardiomyopathy (TTC) have been individually noted to occur in older persons, especially women, following a significant emotional event or physical exertion. In our present case, a 66 year-old female presented to the emergency room with simultaneous occurrence of TGA and TTC following a sexual encounter.

Whether TGA initiates TTC or vice versa remains unclear. However, literature provides evidence of emotional and physicals stressors in both conditions [9]. During emotional arousal, the autonomic system releases stress modulators, cortisol and catecholamines. These specific modulators mediate the hypothalamic-pituitary adrenocortical (HPA) axis and sympathetic-adrenomedullary (SAM) systems, respectively. However, in TGA and TTC, autonomic regulation is altered resulting in catecholamines release for a prolonged period creating negative psychological and physical symptoms including but not limited to headache, hypertension, anxiety and panic attacks. This catecholamine surge creates a neurotoxic 
effect on the hippocampus and over-stimulates adrenergic receptors in the myocardium $[9,10]$.

In addition to changes in autonomic regulation, physical factors have also been noted in TGA and TTC. Physical exertion during strenuous activities can elevate intravenous pressure throughout the body. Increased pressure compromises Internal Jugular Vein (IJV) drainage during the Valsalva Maneuver (VM) ultimately resulting in venous congestion [1].

It is our belief that our patient developed TGA due to physical exertion, in this case sexual intercourse; the psychological stress of TGA, ultimately resulting in TTC.

Established facts: Simultaneous occurrence of TGA and TTC has been seldom published.

Possible pathophysiological mechanisms

Role of catecholamines: Neurotoxic effects on hippocampal function occurring after emotional or physical stress can result in overstimulation of adrenergic receptors in the myocardium, the cerebrum and their vasculatures resulting in a sequence of TGATTC [6]. Whether pre-existing comorbidities such as myocardial compromise can affect the development of the TGA-TTC sequence requires further investigation.

Role of venous congestion pathogenesis: Prior literature states that Internal Jugular Vein (IJV) drainage is relatively compromised during the Valsalva Maneuver (VM) in the patients with TGA. A study correlating TGA and impairment of cerebral venous drainage through an ultrasound investigation concluded that venous stasis and occlusion may cause narrowing of cerebral arterioles, which can further jeopardize cerebral hemodynamics. Particularly, during a VM-like maneuver, an insufficient IJV patency would limit the release of increased intracranial pressure and venous congestion in the basilar plexus and cavernous sinus [1].

Role of physiologic stress as precipitating factors: Significant physical or emotionally straining activities can result in numerous hormonal and autonomic alterations on the human body. A considerable elevation in the systemic intravenous pressure during the Valsalva maneuver increases the possibility of venous congestion with a potential risk for the TGA-TTC sequence [2]. This has been suggested in other reports of the Valsalva maneuver and its association with Valsalva retinopathy secondary to elevation of intravenous pressure resulting in the increased likelihood of retinal hemorrhages [7].

Role of coronary artery disease and coronary vasospasm: Although the clinical presentation imitates that of an acute MI, coronary arteriography does not demonstrate any obstructive lesions. The hypothesis of coronary vascular dysfunction related to multi- focal coronary vasospasm on coronary angiography should still be further investigated [3].

\section{Learning Points}

- Both transient global amnesia (TGA) and Takotsubo cardiomyopathy (TTC) share common features including reversibility, occurrence in women during the fifth and sixth decade and similar precipitating triggers.

- Concomitant TGA and TTC may occur in a subset of older patients secondary to an autonomic surge resulting in cardiotoxicity and cerebral venous congestion [5].

- Patients who present with TGA should undergo complete assessment including a survey of precipitating events and serial ECGs with measurement of cardiac enzymes to monitor for the development of TTC.

\section{Conclusion}

Transient global amnesia (TGA) is considered a primary neurologic disorder however, it is important to recognize a possible relationship between TGA and TTC, in order to manage the cardiac complications that may arise from TTC. Although concurrent occurrence of both TGA and TTC may be a rare phenomenon in comparison to individual presentations, understanding the associated factors and a possible causal relationship between both etiologies may be of clinical significance.

\section{Consent for Publication}

Verbal and written consent was obtained from the patient.

\section{Competing Interests}

The authors have no conflict of interests to declare.

\section{Bibliography}

1. Han K., et al. "Transient Global Amnesia Linked to Impairment of Brain Venous Drainage: An Ultrasound Investigation". Frontiers in Neurology 10 (2019): 67.

2. Mahler DE. “Transient Global Amnesia”. UpToDate (2019).

3. Misumi D., et al. "Coronary spasm as a cause of takotsubo cardiomyopathy and intraventricular obstruction". Journal of Cardiology Cases 2.2 (2010): 83-87.

4. Pyle LM., et al. "Concomitant transient global amnesia and takotsubo cardiomyopathy following a stressful event". Clinical Autonomic Research 28 (2018): 597-598.

5. Quick S., et al. "Transient global amnesia and broken heart syndrome: two faces of one pathology". Clinical Autonomic Research 25 (2015): 189-191. 
6. Reeder DS and Prasad D. "Clinical manifestations and diagnosis of stress (takotsubo) cardiomyopathy". UpToDate (2019).

7. Rubaie KA and Arevalo JF. "Valsalva Retinopathy Associated with Sexual Activity". Case Reports in Medicine (2014).

8. Stollberger C., et al. "Tako-tsubo cardiomyopathy with transient global amnesia and cerebellar embolic stroke triggered by existential fear". Neurologia i Neurochirurgia Polska 52.3 (2018): 394-396.

9. Y-Hassan S and De Palma R. "Contemporary review on the pathogenesis of takotsubo syndrome: The heart shedding tears: Norepinephrine churn and foam at the cardiac sympathetic nerve terminals". International Journal of Cardiology 228 (2017): 528-536.

10. Peggy Quinette., et al. "What does transient global amnesia really mean? Review of the literature and thorough study of 142 cases". Brain 129.7 (2006): 1640-1658.

\section{Assets from publication with us}

- Prompt Acknowledgement after receiving the article

- Thorough Double blinded peer review

- Rapid Publication

- Issue of Publication Certificate

- High visibility of your Published work

Website: www.actascientific.com/

Submit Article: www.actascientific.com/submission.php Email us: editor@actascientific.com

Contact us: +919182824667 